\title{
EDUCATIONAL NEUROSCIENCE FOR SECOND LANGUAGE CLASSROOMS
}

\author{
Siusana Kweldju \\ Universitas Negeri Malang \\ siusana.kweldju.fs@um.ac.id
}

\begin{abstract}
Today's classrooms, including second language classrooms, are created to be more engaging, effective and empowering for learners to develop their knowledge, interests and experiences. One effort is to consider how the brain learns in the classroom; what the brain is able to do, and what is not. Educational neuroscience is a transdisciplinary convergence of neurosciences to translate neuroscience research into classroom practice, including how a second language is learned. Therefore, every teacher, including second language teachers should know about neuroscience. Educational neuroscience is a wide field which still remains open for further investigation. Exploring the latest findings from neuroscience research, this paper proposes seven second language classroom principles. The principles are developed mostly based on research on declarative memory, instead of univcrsal grammar, which is developed based on mentalistic philosophy.
\end{abstract}

Keywords: Educational Neuroscience, Neuroeducation, Second Language Classrooms, Language Acquisition

\section{INTRODUCTION}

Owing to the advancement of computer science and engineering, medical science, projectional radiography, and fiber optic sensing technology, noninvasive techniques have been successful to produce images of the internal aspect of the body, including the brain; especially in measuring brain activities for revealing how the human brain functions. Those instruments can either directly, or indirectly detect neural activity through the changes in blood-oxygenation. Even today, scientists are still not satisfied with the advanced conventional instrumentation and techniques such as fMRI, NIRS, MEG, EEG owing to their limitations in spatial and temporal resolution. They never stop to improve the techniques to produce much more accurate view of the spatiotemporal dynamics of brain activity (Hiwaki \& Miyaguchi, 2018). The little known field of brain activity has rapidly reached the increasingly higher advancement of progress along with the continuous improvement of the neuroimaging techniques. Results of the investigations produce an astonishing wealth of new knowledge about the brain capacity to learn and the way learning occurs in the brain.

As there was limited research on brain mechanism in the past, L2 language instruction was hardly brain-based. Only some implication from SLA research influenced some teachers' instructional principles, for example, the influence of critical period hypothesis. Neurobiological investigation to critical period hypothesis was mostly conducted to infants' early flexibility of brain mechanisms in language acquisition, but not adult second language learners'. Also, due to the limited technology in the past, language network was only understood to take place at the perisylvian cortical regions, namely the Broca's and Wernicke's areas. Today, however, neuroscientists are able to examine the relationship between language tasks and brain activities.

\section{Brain Resarch for Learning}

Educational neuroscience or neuroeducation is a young discipline that translates neuroscience research into classroom practice. It studies cognitive psychology, neuroscience, language and education theory for improving learning and instruction. Language which is represented in current literacy-reading, writing, speaking, listening, thinking and calculating--is understood as a mediating factor to connect the mind and the brain. The mind has been demonstrated to be localizable in the brain, as there is an association of psychological function with particular structures in the brain. Kweldju (2015), for example, has shown that reading is not a straight-forward graph-to-sound decoding mechanism, but it involves subprocesses in different areas and pathways of the brain, including the primitive brain areas, which were thought unlikely for higher cognitive and linguistic functions. This 
leads to the assumption that learning and instructions without considering neurobiological processes or the synaptic firing and patterns, will impair learners' cpacity to function optimally (Robb, 2016).

Neurologically, learning is forming new neural connections and new domains in the memory-a process of changing the brain. Thus, learning is successful when the changes of brain activities are necessary for the proper storage and retrieval of information. Actually the number of neurons or brain cells does not change much throughout life, that is about 100 billion brain cells, because the human infant is born with approximately 100 billion brain cells, or neurons. But what may change dramatically is the tens of thousands of connections among the 100 billion neurons. Studies show that the connections can amount to approximately 1,000 trillion synaptic connections. The creation of the new connections and their continual restructuring and changes in the nervous system are known as neuroplasticity.

In the past, to promote teaching and learning, disciplines such as psychology, sociology and philosophy have contributed a great deal to education theory and practice. In the last 20 years, however, educators begin to realize that they need brain-informed approach to learning. Findings from neuroscience research to understand the mental processes of learning are important for designing instructions which consider how the way the brain learns, and for preparing the brain for learning. Educators need to investigate, for example, how to apply neuroscientists' findings on working memory and long term memory into their students' learning process. Neuroscientists have discovered that the main location of the working memory is the different parts of the prefrontal cortext, while the long-term memory is located in various regions of the cortex, according to the types of the memory. The hippocampus in the medial temporal lobe regions is the transition from the working to the long memory. The regions are connected extensively with temporal and parietal neo-cortical regions (Suzuki \& Arnaral, 1994). It is educational neuroscience which focuses on how to apply neuroscience to education. Based on the findings, instructions should consider the best way of activating those brain regions which are involved in various component skills.

Thereby, effective instructions should make learners maximize the activation of the frontal lobes that connects multiple brain regions. One way to do it is to engage students with open-ended conceptual questions (Watagodakumbura, 2015; Zhou, 2018), and other active learning techniques, such as peer instructions, case studies, creative scenarios, and simulations.

\section{Plasticity in Human Brain}

That the human brain is a learning brain has the extraordinary plasticity to change, to adjust itself with environmental demands. It grows and makes new synaptic connections throughout one's life. On that account, all humans have the ability to learn explicit and implicit cognitive, social and motoric skills through the life span, which is the main neural basis for lifelong learning. This plasticity enables the development and modifications of the function and structure of neural circuits.

The process of synaptic plasticity involves both the synaptic strengthening or weakening between brain cells, and the creation or elimination of neural connections. Synaptic strengthening or weakening can reweight existing circuit connections, while synapse formation and elimination create new connections. This depends on how often and when the connections are activated.

\section{Adult Neurogenesis}

Thousands of brand new neurons can be created each day in a process called adult neurogenesis, which is the process by which nervous system cells, the neurons, are produced by neural stem cells. Studies in the brain of adult mammals have overturned the doctrine of the 20th century that the adult brain has no capacity for generating new neurons. The discovery has even been prospective for the therapeutic target for Alzheimer's disease. As found by Deng et al. (2010), adult neurogenesis, originating from neural progenitor cells (NPCs), has been consistently observed in two regions: the subventricular zone (SVZ) of the lateral ventricle and the subgranular zone (SGZ) of the dentate gyrus in the hippocampus.

This is because the adults' brain contains divisible neural stem cells. However, most of the new cells do not survive after a few weeks. In fact, over half of them, if not more, die within just a few weeks of their birth. One of the most effective ways to keep the cells from dying is by effortful learning to integrate them into the circuitry of the adult hippocampus. The findings by Shors et al. (2012) showed that the new brain cells as the product of neurogenesis will only survive, when the adult learner is involved in some kind of effortful learning experience when the cells are about one week of age. If learning does occur, the new cells become incorporated into the brain circuits used for learning.

Exercise is important to increase the volume of the regenerated hippocampus because neurogenesis needs both stimulation and enriched learning environment. Neurogenesis fails to happen, however, when chronic stress takes place. Baptista and Andrade (in Cremer \& Grilli, 2018) discover that stress leads to a general decrease in the neurogenic function, and it may also leads to maladaptive stress responses that cause anxiety and depression. Depression itself may lead to dysfunction of brain circuits. 


\section{Plasticity and Adult Neurogenesis}

Today, the discovery of neurogenesis leads to the suggestion that learning is not necessary with fun only. Learning should also be challenging, although chronic stress may inhibit neurogenesis. Thus, adults' learning and memory do not only depend on synaptic plasticity or changes in synaptic strength, but also a memory structure called hippocampus-- another type of plasticity for memory formation. Gu et al. (2013) show a current unique evidence that lifelong addition of new hippocampal neurons continuously rejuvenates adult brain. The continuously generated cohorts of neurons by hippocampal dentate gyrus integrate into the existing neural circuit under the regulation of existing global and local neural activity. Recently, it has been discovered that neurogenesis can also take place in amygdala.

\section{The Need of Understanding Neurobiological Basis of Learning}

Neuroscience literacy is important for teachers to influence their practice, and even parents need to know it, as well. If teachers want to reduce the suffering of the classroom, they need to know what happens inside the students (Kelly, 2017); Hart (1978) calls it the brain-compatible teaching. It is true that some findings from brain studies tell teachers what they already know through training, experience and intuitions, although it is without neurological evidence. For example, Bloom's taxonomy of cognitive domains from lower cognitive functions to higher ones consists of remembering, understanding, applying, analyzing, evaluating, and creating--developed without any neurobiological consideration--is now discovered quite parallel with neuroscience findings. Frontal lobes of the neocortex are crucial for the higher order thinking skills, and they become more active when they are involved in conflicting conditions. The frontal lobe is also directly connected with every distinct functional unit of the brain. It navigates attention, stores working memory and supports temporal integration. Today cognitive neuroscience can show how different the more creative and less creative individuals' brain structures. Creative individuals have higher cortical thickness in the right posterior cingulate and right angular gyrus, and they also experience greater bilateral frontal activity. The cortical thinkness of non-creative individuals, in contrast, is found in a region within the lingual gyrus and left lateral orbitofrontal area (Jung et al., 2010).

However, there are a lot of new neurobiological findings that teachers need to know, particularly concerning neurological evidence versus neuromyth. The studies of the living brains discovered that it is not true that: a child should learn one language before learning another; intelligence is fixed; there are two types of brain thinkers--left brain and right brain thinkers; different learners use different learning styles; physical exercise enhances learning; girls and boys have different cognitive abilities; most learning happens in the first 3 years of life.

\section{All Learning Needs Memory}

All learning needs memory. Learning and memory require the formation of new neural networks in the brain. Memory is the storage and retrieval of any form of information, but cognitively it is not a unitory function. Today we know the working and long-term memory, the explicit and the implicit memory.

\section{Storing and Strengthening New Memory: Synapse and Transmitter}

Memories, however, can change, when the strengths of the synaptic connections between neurons change (Poo et al, 2016). This is called synaptic plasticity, or the ability of synapses to strengthen or to weaken over time caused by how they are activated. Synaptic plasticity is one form of neural plasticity that could underlie learning and memory. Memories of an event are stored initially in the hippocampus, where synapses begin to form new circuits that stores a new memory. An increase in the strength of a relatively small number of synapses can bind connected neurons into a circuit that stores a new memory. It becomes stronger when the release of transmitter at a synapse is repeatedly correlated with firing of action potentials in the postsynaptic neuron. In contrast, when the release of transmitter at a synapse repeatedly fails to correlate with postsynaptic firing, they become gradually weaker and may disappear altogther.

From the educational neuroscience perspective, an effective teaching process is the one that can successfully help the strengthening of the students' synaptic connections. It should meet the human brain's ability to process new information to form functional structures aimed at solving problems. Anderson et al. (2018) propose reinforcement-based approaches to studying than the recognition-based information learning task. In this case, the teacher does not only present the information, but leverage reinforcement learning through student-instructor interactions to advance to clarification and elaboration of knowledge.

Language Capacity Depends on Memory Systems: Declarative and Procedural, instead of Language Innate Faculty 
One of Chomsky's hypotheses is the Language Acquisition Device (LAD) and later he revised the idea and named it Universal Grammar (UG); that is the innate language faculty for the rapid language development in human. The hypothesis suggests that the nature of language is neurocognitively domain-specific. The basic postulate of UG is that a certain set of structural rules are innate to humans which governs human language, independent of sensory experience. Children adopt specified syntactic rules that conform to UG, known as mental grammar, as the unconscious, highly abstract grammatical principles that allows a speaker to produce understandable language, known as mental grammar.

However, it has been discovered that language is so diverse and universality is rare. Lin (2017) is one of those researchers who have refuted the hypothesis. Lin criticized Chomsky's methodology which only relies on one language: English, while there were about 6000 existing languages today, and there were about 500,000 languages in human history. The investigation of one language only cannot represent the investigation of thousands of languages.

Lab-based experiments about brain organization associated with language functions show, however, that UG is in contrast to the dclarative/procedural (DP) theory, which posits that the two memory systems play critical roles in the learning, representation, and processing of both non-language and language domains. UG believes that language is domain specific, while DP theory believes that language is domain general. DP is motivated by Desai et al.'s (2007) piggyback model on additional mutations in evolution and biology, that new functions of more fit cells often piggyback on previously existing mechanisms with less fit cells. The DP model posits that since most aspects of language must be learned, language should depend heavily on the declarative and procedural learning and memory systems, since these systems may be the two most important learning systems in the brain.

However, it is important to bear in mind that language is not subserved by nonlanguage systems, but rather both language and nonlanguage functions are subserved in a similar way. That isas mentioned above--most if not all of language must be learned, and declarative and procedural memory systems are the most important systems in the mind. Ullman (2013) shows that language, instead of being domain specific and independent of sensory experience, relies largely on both declarative and procedural memory which interact, cooperate and compete.

The procedural memory--a system that underlies rules and sequences--subserves mental grammar, and is rooted in frontal/basal-ganglia structures, while declarative memory subserves mental lexicon. The procedural memory system is called implicit memory system, for acquiring information on how to perform certain procedures, specially for subconscious knowledge of habit formation through repeated exposure to stimuli, or practice with the skill or habit for consolidating new skills, and for processing skills after being automatized (Ullman \& Lovelett, 2016). Following this, the acquired skills or knowledge is not accessible to conscious memory. The network is rooted in frontal/basal-ganglia circuits, including premotor regions and BA 44 within frontal cortex, and the caudate nucleus within the basal ganglia. It may also include portions of inferior parietal cortex, superior temporal cortex, the cerebellum, and the dorsal stream. Dopamine is the transmitter for procedural learning.

\section{Children and Procedural Memory}

As discussed above, children benefits more from the procedural memory. In fact, all children learn their grammar using their procedural memory, except those with specific language impairment (SLI) who should rely on declarative memory for its compensatory role in their grammatical defisit (Conti-Ramsden et al., 2015).

Next to grammar, procedural memory which deals with implicit learning of new information and underlies the gradual learning of habits and skills controls the learning of speech sound contrasts. Therefore, compared to adults, children learning a second language excell in pronunciation (Quam, 2018).

\section{Principles for Classroom Second Language Learning}

\section{Form Learning, and Habit Formation for Natural Communication Skills}

As restated by Ullman (2005), the declarative memory system comprises episodic memory and semantic memory. Factual knowledge is called semantic knowledge, and eventive knowledge is called episodic knowledge. Thus, semantic memory is the recall of general facts, while episodic memory is the recall of personal facts.

According to Ullman (2005), classroom SLA needs to consider how to make declarative knowledge go closely together with the procedural knowledge through practice. Although declarative knowledge cannot replace procedural knoweldge, but lexical forms and constructions stored in the declarative memory can gradually and implicitly be abstracted by the procedural memory system. In contrast to the declarative memory, the procedural memory system underlies implicit knowledge which is acquired slowly through repeated exposure, but once it is learned the knowledge is processed more rapidly and automatically than knowledge in declarative memory. 
Based on the neurobiological findings above, students need to explicitly involved in form learning to develop their lexical, grammatical, and phonological competence, while simultaneously need the opportunities to freely use those forms and other forms they have learned for developing their natural language communication skills-both receptively and productively, oral and written--with accuracy, fluency and complexity. This idea draws our attention to the focus-on form, focus-on-forms, and focus-on-meaning instructions.

\section{Age and Second Language learning: Maximizing the Compensatory Role of Declarative and Working Memory Capacity}

Along the way, a competitive interaction takes place between the declarative and procedural memory. During early adulthood attenuation is detected at the procedural memory, but enhancement takes place at the declarative memory. It means that older children who learn a second language benefit more from declarative memory system compared to the younger ones. Following this, the acquisition of grammatical-procedural knowledge by adults is expected to be more problematic than the acquisition of lexical-declarative knowledge in young children. The procedural system is subject to early critical period effects. The increasing levels of estrogen during adolescence may inhibit the procedural memory system as well as enhance declarative memory. Therefore, as suggested by Ullman (2005), older children and adolescents learning a second language need to be involved in memorization activities, including in learning grammatical rules.

However, the advantage experienced by adolescents in using declarative memory for developing their lexical knowledge and complex linguistic representations is gradually declining in early adulthood, with more notable losses in old age. One possible reason is the decline of the estrogen levels. Another reason, according to Yousef et al. (2019) seems to be aged blood. As women have more estrogen than men, during adolescence and early adulthood they have the potential to take more advantage of the declarative memory. However, older women, especially after menopause, have difficulty to take advantage of the declarative memory. This could implicate the learning of a second language, but it does not mean that they cannot learn a second language in their advanced age.

Older children and adolescents learning a second language need to be involved in memorization activities, not only for developing their lexico-grammatical skills, but also in learning gramatical rules. Studies have shown how they benefit from their declarative memory in memorizing word-specific knowledge (Ullman, 2004). Further, students need the opportunity to maximize their memory capacity and the compensatory role of memory. Even people with advanced age can improve their learning performance by firstly improving their working memory capacity to increase their attentiveness. In fact, the declarative-memory system relies on working memory and attention (Filoteo et al., 2010).

Working memory is a subpart of short-term memory. It temporarily stores, organizes and manipulates information. Short-term memory is the information that is held in mind for a very short period of time. There is an association between working memory and declarative memory for both encoding and retrieval of information. Today research has shown that working memory capacity can also be improved through adaptive and extended training (Klingberg, 2010).

\section{Life-long Learning is Possible for Second Language Learners}

Adult brains continuously rejuvanates. Therefore, adults can be involved and successful in second language life-long learning. This neurobiological finding cen explain Linhart-Wegschaider's findings (2010) when she splitted up 30 test persons aged 20-69 learning Chinese Mandarin as a second language. She discovered that in terms of overal performance the older learner group outperformed their younger peers. However, in terms of retentiveness the middle-aged group outperforms both their younger and older peers.

\section{Initial Learning Depends on Declarative Memory: Lexically-based Language Learning is Used as an Approach}

As declarative memory system works faster and more effective at the initial stage of learing, lexically-based language learning is best used at the inital stage of second language instruction, and it can even continually be used to the advanced level. Lexically-based language teaching believes that grammar and vocabulary are inseparable because language consists of prefabricated patterns, and collocation is introduced and used very early in learning, as it is the central feature of lexis. Conscious learning, repetition or memorization plays an important role in the process. At the later stage of learning, learners are prepared to develop their autonomous learning for developing their stylistic skill, and their metaphorical skills (Kweldju 1999, 2003a, 2003b, 2004, 2005).

However, since the procedural memory system should also be activated for the acquisition of grammar and and the automatic language skills, learners should also be gradually exposed to the spontaneous use of natural communication, both in real life and semi-real life environment. 


\section{Activating Hippocampus for Testing Effect: Quizzes and Active Learning Techniques for Memory Improvement}

Hippocampus plays an important role in language learning, especially for semantic memory retrieval. Breitenstein et al. (2005) found that the learning of new lexicon depends on the left hippocampus and neocortical regions. Deng et al. (2010) show that adult hippocampal neurogenesis can also be modulated by artificial induction of network activity. It also needs an enriched environment; that is, a larger number of opportunities for learning. Aside from word learning, Alamri (2017) showed that hippocampus is also important for the flexible use of language during real-time processing.

Based on the brain research findings above, second language learning can take place throughout one's life. As language learning needs both episodic and semantic memory, and memory needs repetition. Learning for adults, can only be successful, when the process of learning is through repetition and enriched environment. The learning itself should also be challenging enough or effortful, and should not merely be fun.

As learning should be efforful and relies on memory, and memory includes retrieval processing, classroom activities should also include retrieval practice or Ullman and Lovelett (2016) call it testing effect for improving memory.

Remembering and understanding are more associated with the hippocampus, a satellite organ. Thereby, effective instructions should make learners maximize the activation of the frontal lobes that connects multiple brain regions. One way to do it is to engage students with open-ended conceptual questions (Watagodakumbura, 2015; Zhou, 2018), and other active learning techniques, such as peer instructions, case studies, creative scenarios, and simulations.

\section{Psychological Stress Enhances Semantic Memory: Students Need to Self-regulate their Learning}

It was already discovered that effortful learning is important for adults for keeping new adult neurons. Therefore, teachers are unnecessary to believe that games and fun activities are a vital part of the success of second language instruction in the classroom, especially when they are still not supported by brain research. It is wrong that stress in language learning can cause poor performance. Brain research has discovered that acute psychological stress consistently impairs episodic memory, but it enhances semantic memory, because the two memories differ in their neural substrates.

Although both episodic- and adults' semantic-memory retrieval recruit the hippocampus, and stress impairs the brain regions associated with retrieval including hippocampus, but stress does not disrupt semantic memory. The reason is that the hippocampus is only essential for the retrieval of episodic memories. In contrast, semantic memory retrieval recruits neocortical and striatal pathways, which provide retrieval routes that bypass the hippocampus, as semantic memories can be retrieved via extra-hippocampal pathways. Even retrieval through the neocortical and striatal regions enhances semantic memory. From an evolutionary perspective, the stressed brain adaptively enhances some cognitive processes like attention at the expense of all memory retrieval (Smith et al., 2019).

If teachers do not want to overburden their students, teachers can help students how to regulate their study routines, and how to self-regulate their learning such as learning regularly, repetitively, and not delaying learning.

\section{Vocabulary Learning: Activating both Episodic and Semantic Memory}

Practicing vocabulary can be more successful when it is used both for general knowledge and personal knowledge; that is activating both the semantic memory and episodic memory. At the initial stage students are engaged with activities that can help them to relate the new vocabulary with their own personal experience, especially when they could relate the new words with the objects bearing the names that they are familiar with. And within one week's time they repetitiously practice the words for consolidation, using the words for general knowledge to store the information in the semantic memory. Since learning vocabulary is not only for storing information but also recalling information, episodic memory is still necessary at the later stage of learning, because it is used as the context for recalling events. Context is the organizing and disambiguiting principle of the lexicon. Learning vocabulary needs diversity and distinctiveness of contexts, both from general facts and personal experience (Jones, et al. 2017). Thus, repetition of learning alone is insufficient for semantic learning and communicative behavior,

Neurobiologically, according to a number of research the initial stage of word learning depends on the episodic memory networks with the hippocampus as the critical component. After further activation of consolidation, a shift towards more systematic, lexicalized coding of the memory representation in a distributed neocortical network occurs. Through consolidation, novel words bacome integrated into the existing mental lexicon in the semantic memory. It is after this integration process that interaction between novel words and existing words is observed. Sleep has a role for the integration process (Takashima et al., 2017). 
Recalling also needs the episodic memory. Memory is not only the process of encoding, storing and consolidating information, but also recalling information that has been acquired. According to studies on patients with semantic dementia, for example, they complain about their memory impairment in naming, or retrieving and recalling words and information. Although they can produce fluent and grammatically correct speech, but they suffer from memory loss of words. In fact, one's personal experience stored in the episodic memory also involves one's linguistic experience. Neuroanatomically, on close inspection semantic dementia patients are not only impacted by the degeneration of semantic memory system, but also episodic retrieval in the context of marked hippocampal atrophy. Therefore, their verbal episodic deficit impacted their semantic naming performance, without affecting their visual episodic performance. In other words, their episodic memory deficits were confined to the verbal domain only, and they could still keep their non-verbal episodic memory performance. Episodic memory deficits that arise in semantic dimentia SD appear to be primarily mediated by the conceptual loading of the to-be-remembered stimuli. The regions implicated included the bilateral anterior temporal fusiform cortex, bilateral temporal pole, bilateral orbitofrontal cortices, bilateral lateral temporal cortex, bilateral parahippocampal gyrus, bilateral amygdala, bilateral Native hippocampus, and the left lateral occipital cortex (Irish et al., 2012).

\section{Non-Native Speakers may Reach Native/Native-like Proficiency}

In some cases, non-native speakers may also reach native or native-like proficiency, According Schuman (2006) this is because some people do not have average brain, they might have certain hypertrophies (increase in the volume) in their neural structure that provide advantages in postcritical period second language aquisition. As an extreme example, Einstein had a different brain; he had many more glial cells (support structures for neurons) in all areas studied than normal brain. There was also a missing part in Einstein's brain; that was the Sylvian fissure that made his inferior parietal lobes in two hemispheres 15 percent wider and markedly asymmetrical and more spherical than normal. The vacancy enabled the neurons in the regions for incorporating and synthesizing information from multiple other brain regions.

\section{CONCLUSIONS}

One goal of neurobiological research is to provide new insights how the brain learns. Second language instruction has long adopted theories on linguistics, psychology, psycholinguistics, and sociology to make second language learning more successful. However, most of those theories, including UG, are more speculative without neurological support about how the brain processes language. Today, second language instruction should be brain-based to create effective learning in a shorter period of time. Neuroscience is a sound science to show the actual human nervous system and how it acquires language. As long as language learning is concerned, declarative memory is the most central for older children's and adult's second language acquisition.

Based on neurological evidences, this paper proposes seven principles for classroom second language instruction: (a) both form learning and habit formation for natural communication skills should be started very early in classroom instruction, (b) a second language can be started to learn at any age; although older learners cannot benefit from the procedural memory, they can maximize the compensatory role of the declarative and working memory capacity, (c) since initial classroom second language learning depends on declarative memory, lexically-based language learning is best used as an instructional approach, (d) as testing effect is the preferable goal for activating the hippocampus, quizzes and active learning techniques are best for memory improvement, (e) since psychological stress enhances semantic memory, students need to self-regulate their learning, (f) in order that vocabulary learning is successful, both episodic and semantic memory should be activated, and (g) non-native speakers have also the possibility to reach native and native-like proficiency, especially because people may have different brains.

\section{REFERENCES}

Alamri, S. (2017). The Contribution of the Hippocampus to Language Processing. (Unpublished doctoral dissertation). Universitas de Barcelona. Barcelona, Spain.

Andersen, S.J., Hecker, K.G., Krigolson, O.E. \& Jamniczky, H.A. (2018). A Reinforcement-based Learning Paradigm Increases Anatomical Learning and Reention-A Neuroeducational Study. Frontiers in Human 12(38).

Breitenstein, C., Jansen, A., Deppe, M. Foerster, A.F., Sommer, J., Wolbers, T., Knecht, S. (2005). Hippocampus activity differentiates good from poor learners of a novel lexicon. Neuroimage, 25(3), pp. 958-68. 
Conti-Ramsden, G., Ullman, M.T., Lum, J.A.G. (2015). The relation between receptive grammar and procedural, declarative, and working memory in specific language impairment. Frontiers in Psychology. Accessable at https://doi.org/10.3389/fpsyg.2015.01090

Cremer, H. \& Grilli, M.G. Adult Hippocampal Neurogenesis: Regulation and Possible Functional and Clinical Correlates (Review of the book by Baptista, P. \& Andrede, J.P.) Frontiers in Neuroanatomy, $\quad 5 \quad$ June 2018. 201https://www.frontiersin.org/articles/10.3389/fnana.2018.00044/full

Deng, W., J.B. Aimone \& F.H. Gage, 2010. New neurons and new memories: how does adult hippocampal neurogenesis affect learning and memory? Nature Reviews Neuroscience, pp.1-12.

Desai, M.M., Fisher, D.S., andMurray, A.W. (2007). The speed of evolution and maintenance of variation in asexual populations. Current Biology, 17, pp.385-39.

Filoteo J. V., Lauritzen S., Maddox W. T. (2010). Removing the frontal lobes: the effects of engaging executive functions on perceptual category learning. Psychological Science. 21, pp. 415-23.

Gu, Y., Janoschka, S., Ge, S. (2013). Neurogenesis and hippocampal plasticity in adult brain. Current Topics in Behavioral Neurosciences, 15, pp. $31-48$.

Hart, L. (1978). Brain-compatible teaching. Today's-Education, 67, pp. 42-5.

Hiwaki, O. \& Miyaguchi, H. 2018. Noninvasive measurement of dynamic brain signals using light penetrating the brain, PLOS One (the Public Library of Science One), pp. 1-8.

Irish, M.; Bunk, S.; Tu, S., Kamminga, J.; Hodges, J.R.; Michael Hornbergerc, M.; Piguet, O. (2016), Preservation of episodic memory in semantic dementia: The importance of regions beyond the medial temporal lobes. Neuropsychologia, 81, pp.50-60.

Jones, M.N.; Dye, M.; Jones, B.T., (2017). Context as an organizing principle of lexicon. Psychology of Learning and Motivation, 67, pp.239-83.

Jung, R.E., Segall, J.M., Jeremy Bockholt, H., Flores, R.A., Smith, S.M., Chavez, R.S., \& Haier, R.J. (2010). Neuroanatomy of creativity. Human Brain Mapping, 31(3), pp.398-409.

Kelly, C. 2017. The BrainStudies Boom: Using Neuroscience in ESL/EFL Teacher Training. In T.S. Gregerson \& P. Machtyre, Innovative Practices in Language Teacher Education: Spanning the Spectrum from Intra- to Inter-personal Professional Development. New York: Springer, pp.79102.

Klingberg, T. (2010). Training and plasticity of working memory. Trends in Cognitive Sciences, 14(7), pp.317-24.

Kweldju, S. 1999. English department students' collocation abilities. TEFLIN Journal, 10(1), pp.48-62.

Kweldju, S. 2003a. After vocabulary size, ket's include collocation: a missing link in EAP courses. Jurnal Teknologi Pembelajaran, 11(2), pp. 45-55.

Kweldju, S. 2003b. Improving a college writing instruction: developing L2 learners' awareness and strategies to attain near native stylistic skill. Jurnal IImu Pendidikan, 10(1), pp. 214-36.

Kweldju, S. 2004. The neuropsychological basis of lexically-based language teaching. TEFLIN Journal, 15(1), pp. 74-89.

Kweldju, S. 2005. Lexically-based language teaching: metaphor for enhancing learning. Indonesian JELT, 1(2), pp. 52-65.

Kweldju, S. 2015. Neurobiology research findings: how the brain works during reading. PASAA: Journal of Language Teaching and Learning in Thailand, 50(2), pp. 125-142.

Lin, F.Y. (2017). A refutation of universal grammar. Lingua, 193, pp.1-22.

Linhart-Wegschaider, M.H. (2010). Foreign Language Learning and Advanced Age Age-related aspects and learning results of different adult age groups of autodidactic foreign language learners An empirical investigation. (Unpublished doctoral dissertation). University of Vienna. Wien: Austria.

Poo, M.M., Pignatelli, M., Ryan, T.J., Tonegawa, S., Bonhoeffer, T., Martin, K.C., Rudenko, A., Tsai, L.H., Tsien, R.W., Fishell, G., Mullins, C., Gonçalves, J.T., Shtrahman, M., Johnston, S,T., Gage, F.H., Dan, Y., Long, J., Buzsáki, G., Stevens, C. (2016). What is memory? The present state of the engram. BioMed Central (BMC) Biology, 40, pp.1-18.

Robb, B.E. (2016). A Paradigm Shift in Classroom Learning Practices to Propose Methods Aligned with a Neuroeducation Conceptual Framework. (Unpublished doctoral dissertation). University of Portland, Portland, USA. 
Quam, C., Wang, A., Maddox, W.T., Golisch, K., Lotto, A., (2018). Procedural-Memory, WorkingMemory, and Declarative-Memory Skills Are Each Associated With Dimensional Integration in Sound-Category Learning, Frontier in Psychology, 9, pp.1-15.

Schumann, J.H. (2006). Summing up: Some Themes in the Cognitive Neuroscience of Second Language Acquisition. Language Learning Journals, 56(1), pp.313-9.

Shors, T.J., Anderson, L.M., Curlik, II., Nokia, S.M., (2012). Use it or lose it: How neurogenesis keeps the brain fit for learning. Behavioral Brain Research, 227(2), pp. 450-458.

Suzuki, W. \& Amaral, D.C. (1994). Perirhinal and parahippocampal artices of the macaque monkey: cortical afferants. Journal of Comparative Neurology, 350, pp. 497-533.

Smith, A.M.; Hughs, G.I., Davis, F.C., Thomas, A.K., (2019). Acute stress enhances general-knowledge semantic memory. Hormones and Behavior 109, pp. 38-43.

Takashima, A; Bakker, I; van Hell, J.G.; Janzen, G., McQueen, J.M. (2017). Interaction between episodic and semantic memory networks in the acquisition and consolidation of novel spoken words, Brain and Language, 167, pp.44-60.

Ullman M. T. (2004). Contributions of memory circuits to language: the declarative/procedural model. Cognition, 92, pp. 231-70.

Ullman, M.T. (2005). A cognitive neuroscience perspective on second language acquisition: the declarative/procedural model. JOUR, pp.141-178.

Ullman, M.T. (2013). The role of declarative and procedural memory in disorders of language. Linguistic Variation, 13(2), pp.133-54.

Ullman, M.T. \& Lovelett, J.T. (2016). Implications of the eclarative/procedural model for improving second language learning: The role of memory enhancement techniques. Second Language Research, 34(1), pp. 39-65.

Watagodakumbura, C. (2015). Some Useful Pedagogical Practices: Educational Neuroscience Perspective. Journal of Studies in Education, 5 (4), pp. 191-221.

Yousef, H., Czupalla, C.J., Lee, D., Chen, M.B., Burke, A.N., Zera, K.A., Zandstra, J., Berber, E., Lehallier, B., Mathur, V., Nair, R.V., Bonanno, L.N., Yang, A.C., Peterson, T., Hadeiba, H, Merkel, T., Korbelin, J. Schwaninger, M. Quake, S., Butcher, E.C., Wyss-Coray, T. (2019). Aged blood impairs hippocampal neural precursor activity and activates microglia via brain endothelial cell VCAM1

Zhou, K. (2018). What cognitive neuroscience tells us about creativity education: A literature review. Global Education Review,5(1), pp. 20-34. 